\title{
When does food refusal require professional intervention?
}

Terence M. Dovey ${ }^{a_{*}}$., Claire V. Farrow ${ }^{\mathrm{a}}$., Clarissa I. Martin ${ }^{\mathrm{b}}$., Elaine Isherwood ${ }^{\mathrm{c}}$. \& Jason CG Halford ${ }^{\text {. }}$.

${ }^{\mathrm{a} C e n t r e ~ f o r ~ R e s e a r c h ~ i n t o ~ E a t i n g ~ D i s o r d e r s ~(L U C R E D) ~}$

Department of Human Sciences,

Loughborough University,

Loughborough,

Leicestershire,

LE11 3TU,

UK

${ }^{\mathrm{b}}$ Paediatric Psychology Service,

Shugborough Ward,

Staffordshire General Hospital,

Stafford,

Staffordshire,

ST16 3SA,

UK

${ }^{\mathrm{C}}$ Nutrition and Dietetic Services,

Stafford Central Clinic

North Walls

Stafford

ST21 6DS

UK

${ }^{c}$ Kissileff Laboratory for the Study of Human Ingestive Behaviour, School of Psychology, University of Liverpool,

Eleanor Rathbone Building,

Bedford Street South,

Liverpool

L69 7ZA,

UK

*Author for correspondence. Contact Terry Dovey on t.m.dovey@lboro.ac.uk 


\section{ABSTRACT}

Food refusal can have the potential to lead to nutritional deficiencies, which increases the risk of a variety of communicable and non-communicable diseases. Deciding when food refusal requires professional intervention is complicated by the fact that there is a natural and appropriate stage in a child's development that is characterised by increased levels of rejection of both previously accepted and novel food items. Therefore, choosing to intervene is difficult, which if handled badly can lead to further food refusal and an even more limited diet. Food refusal is often based on individual preferences; however, it can also be defined through pathological behaviours that require psychological intervention. This paper presents and discusses several different types of food refusal behaviours; these are learningdependent, those that are related to a medical complication, selective food refusal, fear-based food refusal and appetite-awareness-autonomy-based food refusal. This paper describes the behaviours and characteristics that are often associated with each; however, emphasis is placed on the possibility that these different types of food refusal can often be co-morbid. The decision to offer professional intervention to the child and their family should be a holistic process based on the level of medical or psychological distress resulting from the food refusal.

\section{KEY TERMS}

Feeding Disorders; Food Refusal; Dietary Variety; Food Neophobia; Picky, Fussy, Choosey Eaters; Selective Eaters; Tube Feeding; Infantile Anorexia; Autism. 


\section{INTRODUCTION}

A wide array of terms have been used to describe feeding disorders in childhood, these include, but are not limited to, picky eating, poor appetite, food refusal, fussy eating, perseverant feeding, neophobia, food phobia, and infantile anorexia. Often the same terms are used to describe different behaviours, and at the same time, different terms can be used to describe the same behaviours. To date, there has been a limited amount of literature attempting to delineate between these different terms and behaviours. This paper attempts to bridge this gap by describing some of the behaviours and symptoms that are associated with what we define as different types of food refusal in childhood. The term food refusal rather than picky or fussy eating is used to describe and distinguish these feeding related behaviours. This deviates somewhat from a common tendency in the literature to distinguish between food refusal and picky eating. We have chosen the term food refusal because all of the feeding behaviours of interest referred to here, whether transient or longer-lasting, involve some level of refusing food.

Despite the large amount of research interest in eating disorders in adults, comparatively little research has explored the causes and consequences of feeding problems and disorders in children. The prevalence rates of anorexia nervosa and bulimia nervosa in the general population are around $0.3 \% \%^{1,2}$ and $1.1 \%^{3,4}$ respectively. In contrast, the prevalence rates of feeding problems in children are much higher. Feeding problems are estimated to affect around $25 \%$ of children at some point in their early lives ${ }^{5}$. Prevalence rates vary considerably according to how feeding problems are defined and reported. The incidence of feeding problems can be elevated when reported by caregivers (50.9\% reporting food refusal at 11 months ${ }^{6}$ ), but appears to be much lower when they are identified by health care professionals (1.4\% identifying food refusal, vomiting and colic between 3 and 12 months $^{7}$ ). Prevalence rates are further increased if other developmental disorders are also considered, as around $80 \%$ of children with disorders associated with cognitive delay exhibit feeding disorders ${ }^{8}$. Feeding problems can have serious consequences for child growth and development, for example they can lead to growth faltering which can be associated with cognitive impairment ${ }^{9}$. Moreover, although there has been relatively little longitudinal research on the topic, the research to date does suggest that feeding disorders in early life may predict the later development of eating disorders ${ }^{10}$.

TABLE 1 GOES HERE.

The DSM-IV provides the diagnostic criteria for feeding disorders in infancy or early childhood (see Table 1). Both the diagnostic criteria and associated descriptive features of feeding disorders are notably ambiguous. Although this ambiguity may be useful to allow flexibility in the application of the criteria to a vast array of feeding related problems, this ambiguity has also allowed for an array of interpretations of the DSM-IV criteria resulting in a confused and 
equally ambiguous field of research. For example, the DSM-IV associated descriptive features section states that:

\begin{abstract}
"Infants with feeding disorders are often especially irritable and difficult to console during feeding. They may appear apathetic and withdrawn and may also exhibit developmental delays. In some instances, parent-child interaction problems may contribute to or exacerbate the infant's feeding problem (e.g. presenting food inappropriately or responding to the infant's food refusal as if it were an act of aggression or rejection)." (pg 98)
\end{abstract}

This three sentence description of the disorder suggests a multitude of potential factors that could both cause and exacerbate food refusal. The second sentence in particular could easily be attributed to an autistic spectrum disorder. Furthermore, the use of the word "irritable" covers a multitude of potential contributory factors to food refusal behaviours such as temperament, anxiety, personality, and emotionality as aspects to the rejection of the food item presented. To remain practical and flexible the diagnostic criteria must allow interpretation otherwise it could become redundant; however, it also allows the potential for attribution and publication of a variety of terms that partially or completely overlap with preexisting categorisations of food refusal.

Perhaps as a consequence of this ambiguity, there is little standardisation in the literature of the terms used to define child feeding problems or disorders. This incongruent identification of psychological phenomenon in children's food refusal leaves the field in a state of confusion about what actually constitutes disorder or distinguishes disordered behaviours from normal behaviour. We have previously discussed this to a limited degree in non-clinical samples exploring food neophobia and picky/fussy eating ${ }^{11}$; however, this paper will extend much further to discuss a more comprehensive range of food refusal behaviours. This paper begins by creating "umbrella" terms based on previously published classifications of child food refusal. We then discuss and describe the behavioural characteristics that are often associated with each type of food refusal, and the associated behaviours that may require some form of professional intervention. Following the creation of these categories, they will then be evaluated based on published literature and presented in the order of their potential severity to long-term dietary variety.

\title{
Grouping the Various Terms for Food Refusal
}

Food refusal has been defined previously as the refusal of food at least once a day for a period of one month or more ${ }^{7,12}$, on the DSM-III-R criteria for eating disorders not otherwise specified (EDNOS) ${ }^{8}$ or even by the refusal of all foods ${ }^{13}$. Currently, there is no single widely used definition of what constitutes food refusal and there has been little published discussion about the meaning of this term within the literature on child eating. Despite this, we are not the first authors to attempt to categorise food refusal behaviours. Chatoor \& Ganiban ${ }^{14}$ present a potential grouping strategy for food refusal that has both theoretical grounding and 
practical application. Within their paper, they offer three umbrella terms of unpredictable food refusal, selective food refusal, and fear-based food refusal. They then move on to discuss the clinical description and psychological treatment of one of the terms defined within each category. These are infantile anorexia, selective food refusal and sensory food aversions and post-traumatic feeding disorders. A full diagrammatic representation of these groups is presented in Figure 1. Although this represents an accurate depiction of the potential umbrella terms within children's food refusal, there is some ambiguity within these terms that does not reflect what is often observed in clinical practice. The grouping of terms offered in this paper extends upon those offered by Chatoor \& Ganiban ${ }^{14}$. Notably, these include the extension and separation of the term selective food refusal from learning-dependent (natural) food refusal and the addition of medical complications related food refusal.

Figure 1 GOES HERE.

The literature on pathological aspects of food refusal mainly derives from case study reports in which the authors offer practical guidance on a successful intervention with one or few patients ${ }^{13,15-18}$. Published papers on food refusal that are not based on single or few case studies often fall into one of three camps. These are 1] the child has been diagnosed with a previous medical complaint that directly or indirectly results in the food refusal ${ }^{19,20}$. This means that the children do not meet the DSM-IV diagnostic criteria for feeding disorders (section B) and therefore should not be included in any categorisation of a totally psychological related food refusal. 2] The study sample is taken from a long-term exploration of previous cases within a clinic where the population has received or will be receiving medical attention (e.g., Chatoor et $\mathrm{al}^{21}$ ). These papers offer insight into behaviours associated with extreme or total food refusal; although the length of time the child has been in treatment and the type of treatment the child is undergoing may well influence their eating behaviours. 3] The final group of papers are those based on samples drawn from the general population and therefore reflect variation in normal eating behaviours exhibited by those outside of a clinical setting. Frequently, this type of study explores the concept of learningdependent food refusal within childhood $22-26$.

The large distinction that exists between parentally reported and clinically diagnosed feeding problems suggest that there are a large number of children and families experiencing feeding problems, which are often associated with great anxiety and concern ${ }^{27}$, but remain outside of the clinical threshold for treatment. Moreover, the incorporation of general population studies into the potential defining aspects of food refusal suggests that there should be a separation of the previous umbrella categories to allow for a better description of the phenomena of refusing food. In addition to those offered by Chatoor \& Ganiban ${ }^{14}$, incorporation of two other terms may allow for a better description of current research findings. The first additional category would be food refusal originating or resulting from medical complications or 
procedures. This group would include medical disorders stemming from the fields of genetics, rhinology, laryngology, and gastroenterology. Diseases affecting the major organs are likely to generate food refusal and some minor disease processes can also have a major impact on food acceptance ${ }^{28}$. Specifically, children who have been on prolonged periods of tube-feeding would fit firmly into this category. The second additional category would reflect learning-dependent aspects to food refusal. In essence, this is the natural developmentally dependent food refusal observed in the general population. Terms such as food neophobia and novel sensory food refusal would fit within this category more accurately than within the category of selective food refusal, which accounts for picky, fussy and choosy eaters or those with a moderately limited diet. Although the concepts of food neophobia and picky eating share similar characteristics, they are distinct from each other and require different management strategies ${ }^{29}$. This makes the description and delineations of specific types of food refusal essential, as this will have implications for potential treatment strategies and may even identify individuals who will overcome their food refusal without the need for intervention.

Figure 2 GOES HERE

\section{Diagnosis}

Deciding which children require professional attention for feeding disorders is a complex and holistic process that often does not include the psychologist, dietitian or speech and learning therapist (SALT) who will be actually implementing the therapy. Most feeding clinics in the UK function through a referral system whereby the general practitioner, health visitor, specialist consultant medic or community nurse identify individual children who are experiencing extremely poor dietary variety, growth faltering (also termed failure to thrive), or are experiencing feeding problems that have not responded to primary management strategies. Furthermore, the referral of the child via the general practitioner is often initiated by the parent and thus there is often little independent observation or diagnosis of the child's actual eating behaviour. Patients within clinic can be drawn from any one of the five groups shown in Figure 2 above. This means that within the majority of waiting rooms of feeding clinics in the UK there are likely to be children with: simple yet an extreme form of food neophobia; food phobics; picky/fussy/choosy eaters (selective food refusers, although they are likely to reject most foods); undiagnosed children with autism; diagnosed autistic spectrum disorders; appetite-awareness and/or autonomy-based food refusal; rumination disorder; atypical feeding disorders; and those with current and previous medical conditions. Moreover, these conditions are not necessarily diagnosed in isolation and it is possible that the child may suffer from a combination of these conditions.

Parents and caregivers have an important role in the initial diagnosis and referral of a feeding problem, as they often have access to information about the child's diet and food refusal behaviours that would take time for practitioners to observe. However, often by the time the 
child has been referred to a feeding clinic, parental anxiety can be very high and this can unintentionally exacerbate the food refusal. Parental anxiety can be commonplace in dyads experiencing food refusal, and this is generally a natural response to the distress of parenting a child who is consistently refusing food, and the anxiety evoked by fear of malnutrition in the child $^{30}$. Parental anxiety about their child's eating and weight can be transmitted to the child around mealtimes and food, which, in turn, can elevate the child's anxiety and food refusal. Moreover, parental anxiety can often lead to the use of pressure to eat or force feeding at mealtimes, which can further exacerbate the rejection of foods (e.g., Galloway et $\mathrm{al}^{31}$ ).

In order to differentiate between specific types of food refusal it is pertinent to first explore the literature in order to identify behavioural and observable characteristics that are indicative of specific sub-types of feeding disorder.

\section{The Evidence}

\section{Learning-dependent Food Refusal.}

Characteristic components of learning-dependent food refusal are neophobia, exposure and learning. In essence, the rejection of the food offered is completely dependent on the child's experience with it. This lack of experience combined with natural human tendencies of distrust towards novel stimuli means that the child may initially reject the food item upon the first few presentations ${ }^{32}$. Food rejection based on novelty has been extensively researched within the literature since the late 1970's and has been termed food neophobia ${ }^{33,34}$. Food neophobia is defined as the reluctance to eat, or the avoidance of, new foods ${ }^{35}$ and derives from Rozin and Vollmecke ${ }^{36}$ postulations that humans have evolved behavioural mechanisms that help avoid poisoning.

The characteristic behaviours so far identified as constituent components to food neophobia have indicated that repeated, frequent, prolonged and positive exposure is integral to overcoming these natural human tendencies ${ }^{37}$. There also appears to be a large age dependent effect on this type of food refusal. Current research evidence indicates that food neophobia is very low around the age of weaning and then steadily increases alongside mobility until it peaks between 2 and 6 years of age $\mathrm{e}^{38-40}$. It then rapidly decreases until reaching an individually determined baseline in adolescents ${ }^{41-43}$. Evidence for this psychological construct has been derived from samples of the general population and neophobia usually naturally reduces during the developmental process. In adults, additional personality characteristics have been associated with the severity of food neophobia. These include sensation-seeking ${ }^{44}$, trait anxiety ${ }^{45}$, openness ${ }^{46}$, neuroticism ${ }^{47}$ and general extroverted behaviours.

Another pertinent defining characteristic of food neophobia is that it can be overcome by social influence. It has been consistently observed that parental modelling ${ }^{35}$, parental style ${ }^{48}$, 
parental encouragement ${ }^{49}$, negative comments ${ }^{50}$ and peer group influence ${ }^{51}$ can all affect willingness to try a novel food item. Therefore, positive social models to emulate coupled with positive social encouragement will provide a potent combination to overcoming distrust of a novel food item. In addition, the whole process of accepting novel foods is improved by social facilitation, which suggests that the more people present, and providing positive and suitable models for the child, the increased likelihood that the child will try the new food offered ${ }^{52}$. This social manipulation of food refusal would correspond with Rozin's evolutionary belief that the more people around the child who are trying the food, the less of a perceived threat the food inherently contains.

The combination of the factors mentioned above characterise what constitutes normal food refusal and eating behaviour in children. Therefore, if a child refuses a new food, including any food that has been offered less than fifteen times, has an age dependent variation with peaks between 2 to 6 years, and their food intake is readily manipulated by positive social pressure, then the food refusal should be considered a normal part of the child's development. In addition, it would be expected that the child does not have any developmental delay or problems in controlling their fine motor skills. Based on these premises, it should be assumed that significant qualitative deviations from these overt behaviours would define disordered behaviour needing professional intervention.

\section{Medical Complications-Related Food Refusal}

Various medical complications can result in differences in food refusal, food choice and the amount of food consumed. These medical complications can derive from a variety of ailments from anatomical abnormalities to genetic disorders. Children who share behaviours related to this overarching category are quite difficult to categorise, as they may often have a combination of psychological and medical problems influencing their eating behaviour ${ }^{53}$. Field et $\mathrm{a}^{54}$ has offered an initial categorisation of medical conditions previously associated with feeding and growth problems that are: 1] cardiopulmonary conditions (congenital heart disease, bronchopulmonary dysplasia and asthma); 2] anatomical anomalies (cleft palate, tracheooesophageal fistula and microgastria, which may lead to aspiration); 3] neurological (seizure disorders, traumatic brain injuries, brain tumours, brain malformations, cerebral palsy and developmental disabilities); and 4] renal disease. Field et $\mathrm{al}^{54}$ found that significantly more children with anatomical anomalies had food refusal than children without anomalies and that children with cardiopulmonary conditions had a significantly higher prevalence of dysphagia (a condition that results in problems with swallowing leading to a learned pattern of avoidance) than children without cardiac or pulmonary problems. Nausea, vomiting, poor feeding and a preference for liquids over solids were also reported as symptoms found among children with renal disease. The characterisation and discussion of the eating behaviours associated with medical diagnoses is beyond the scope of this paper (see Harris et $\mathrm{al}^{28}$ and Field et $\mathrm{al}^{54}$ ); rather, this paper focuses on the development of food refusal behaviours which emerge as a consequence of these medical complications. 
Many children are exposed to tube-feeding as a result of certain long-term medical complications. Such conditions include, but are not limited to, gastrointestinal, neurological, oncological and genetic (e.g., cystic fibrosis) conditions ${ }^{55-60}$. Children who are fed by gastrostomy tube are often reported to be much happier and more active, as they can receive a more consistent and adequate energy supply without interference from their medical ailment ${ }^{61}$. Prolonged usage of tube-feeding, especially in younger children, may have consequences that will eventually require psychological intervention. Developmental delay is a common associate of tube-feeding ${ }^{62}$, as are complications with oral motor control and the ability to masticate. The child therefore may require professional help that will include interventions aimed at improving fine motor control, speech, as well as eating behaviour.

Post tube-feeding, food refusal can manifest because of a negative experience during tubefeeding (e.g., repeated nasogastric tube repassing, which may then lead to a choking phobia: see fear-based food refusal), or a result of a lack of developmentally appropriate personal experience with food and eating beyond the liquid form delivered through the nasogastric/gastrostomy tube. This inexperience may result in the child not being able to differentiate between food and non-food items resulting in an increased likelihood of pica type disorders - the consumption of non-nutritive substances. These children will not necessarily show signs of distress around food; rather they may be ambivalent or impassive about food irrespective of its novelty. It is sometimes observed in the clinic that children who have been tube-fed over a long period of time will have little understanding of actual food or appropriate eating behaviour. For example, we have observed that when asked to pretend to feed their teddy bear, many tube-fed children will attempt to tube-feed rather than offer food to the bear's mouth. Tentative inferred evidence suggests that around $10 \%$ of children who are tube-fed may require additional support to get them to eat properly ${ }^{63}$. Evaluation of the potential prevalence rates of subsequent feeding problems associated with tube-feeding merits investigation.

Medical complication-related food refusal also includes ailments related to or causing regurgitation, aspiration or rumination. Aspiration is defined as the inhalation of food or gastric juices into the lungs. It is a complication that results from, but is not specific to, tubefeeding and conditions that result in lower levels of consciousness; however, it is also observed in some young infants. Various practical solutions can be employed to limit aspiration brought about by medical complications. Interested readers should refer to Metheny et $\mathrm{al}^{64}$ for a review. Rumination is usually defined as the repetitive regurgitation of recently ingested food with little or no effort or gagging, whereby the food is then rechewed and reswallowed or vomited ${ }^{65}$. Rumination is a common behaviour associated with a variety of mental disorders and is also prevalent in young children. Overall, this condition is rare outside of developmental disorders and very young children; although further research is 
required in order to determine the epidemiology and associative characteristics in the general population $^{66}$. It is important to remain vigilant to the presence of ruminating behaviours, as it may be likely that individuals who are ruminating will also have additional problems with eating that are probably associated to negative conditioning brought about by their disorder.

Food refusal brought about by the consequence of medical complications can also derive from the treatment strategy. For example, children given a course of chemotherapy will suffer from bouts of nausea and vomiting, which they may inadvertently relate to specific foods, tastes or textures leading to a potent form of food aversion and thus refusal. Weight loss and malnutrition are frequent co-morbidities of chemotherapy ${ }^{67}$ and this treatment regime has been shown to cause food aversions in adults ${ }^{68}$ and children ${ }^{69}$. Therefore, it is important that the therapist considers the treatment strategy as well as the child's illness, as both can have implications for the presence or absence of clinically significant food refusal behaviours.

A multitude of illness-related symptoms will lead to the child not feeling hungry and/or refusing food. Pathologies of any of the major organs, for example resulting in nausea, vomiting, pain or rumination can affect a child's motivation to eat. Management strategies within the treatment process may also affect food refusal too (e.g., tube-feeding). Irrespective of the primary cause of medical-related food refusal, it is quite likely to be responsive to positive social pressure, social facilitation and social influence after a suitable degree of recovery. One important aspect of this process is to placate the caregiver's anxieties about the therapeutic intervention. It is quite likely that the caregivers will be apprehensive about the child eating for the first time after a period of nasogastric feeding or other illness and this inadvertent anxiety has the potential to negatively influence the child's eating behaviour.

\section{Selective Food Refusal.}

Children who fall into the category of selective food refusal are likely to show signs of the normal behaviours expressed in learning-dependent food refusal; however, there are likely to have additional problematic behaviours that result in a further increase in food refusal and thus a decrease in dietary variety. This decrease in dietary variety may be low enough for the child to suffer from significant nutritional deficiencies. Dietary intake will need to be altered in order to maintain sufficient growth and cognitive development ${ }^{70}$. These children are likely to rely heavily on developmental hedonic food choices based on sweet and salt taste preferences and will often avoid bitter or sour tastants. The diet of these children often contains the notable absences of foods high in vitamins, minerals and fibre ${ }^{71}$. Consistent selection of foods lower in fibre often leads to digestive problems, which can further compound the child's eating behaviour and may provide significant negative feedback and inappropriate associations with food selection ${ }^{72}$. Pain resulting from constipation, due to the lack of fibre in the diet, may provide additional impetus for the child to avoid food. This can lead to the child rejecting a much wider range of foods irrespective of whether they have had experience with them or not. Whole food groups are often rejected by the selective food 
refuser making the exposure to new foods more difficult and getting the child to accept most foods problematic. Therefore, mere exposure to food will often have little impact on food selection and acceptance in these children.

One important common characteristic of children with selective food refusal is their tendency to be tactile defensive ${ }^{69}$. Tactile defensiveness is characterised as an overreaction, or offence, resulting in withdrawal from the sensation of being touched, either by another person, or by something in their environment, which most would consider inoffensive ${ }^{73}$. This is exemplified by Chatoor \& Ganiban ${ }^{14}$ who suggest that children who are selective eaters will also exhibit distress if they come into contact with unexpected stimuli such as walking on sand or grass. These authors also suggest that these children may have other sensory sensitivities for odours and sounds. Frequently, these children will be identified for assessment of Autism Spectrum Disorder (ASD) and very often they will return to the clinic with a diagnosis of autistic like behaviours but do not necessarily meet the full criteria for diagnosis. Therefore, these children may have some of the sensory sensitivities of ASD but lack other defining characteristics.

Although children with ASD may also present with significant feeding problems as part of their general psychopathology, food refusal in this group of children is associated with this disorder rather than being a defining feature of it. Children with ASD usually have additional and seemingly illogical 'rules' around what constitutes an acceptable meal, which their parents may come to recognise and will modify their mealtime structure accordingly. Children with ASD constitute a separate group due to the variety of characteristics these children often have beyond their food refusal. They often refuse food based on both fear and sensory properties ${ }^{74}$, which will require a combined approach to overcoming their food refusal. The combined characteristics of the food refusal common to ASD was typified by Cumine et $\mathrm{al}^{75}$ who suggested that concentration on detail, sensory impairments and problems in social compliance come together to define the rejection of foods in children with ASD.

Any associated sensory sensitivity of children who are selective food refusers may have additional unwanted consequences for the child in terms of their food selection. Tactile defensive behaviours in the child may mean that they have sensitivities to oral touch. Therefore, these children are likely to refuse food for additional reasons beyond the normal inexperience related distrust. Rejection characteristics may extend further into the realm of the texture of food ${ }^{13}$. This texture stimulus, determined by the mouth or the hand, will inform decision-making of whether to continue eating or even try the food. Furthermore, this decision-making process will be attenuated by individual differences, thus it is not always possible for the professional to predict which foods a child will refuse prior to observation. In short, one child may refuse foods of one or more specific textures (e.g., soft, crunchy) while another may have a completely different texture based food refusal. Indeed, the texture may 
even become the primary selection criteria above and beyond the actual taste of the food. This hypersensitivity and defensive approach to touch may also mean that the child is more likely to globalise the experience. Here, the child may try one food item and then transfer the experience onto all food items that share similar visual features. For example, an experience with a food that is round and green may mean that all foods that are round or green will be rejected in the future, as the perceptually negative experience of the tried food can be carried over to future exposure episodes.

There are some additional characteristics of selective food refusal that are also frequently observed. Developmental delay can often lead to high instances of selective food refusal ${ }^{76,77}$. At this stage, it is important to differentiate those children that have medical problems, especially with muscle tone, alongside developmental delay, as it is common that these factors co-exist. If the individual has such co-morbidities then it would be pertinent that a medic is involved in the treatment process. Furthermore, there is an increased likelihood that children with selective food refusal will exhibit signs of, or be diagnosed with, other behavioural disorders. Initial indicative behaviours of cyclothymia (a mood disorder similar in nature to a very mild form of bipolar disorder) or oppositional defiant disorders will have a large effect on food refusal and eating behaviour ${ }^{78,79}$.

In summary, children who exhibit behaviours associated with selective food refusal are characterised by a very low dietary variety that is not usually dependent on experience. These children are likely to be tactile defensive and exhibit signs of other developmental and affective disorders without actually meeting the disorders full criteria (e.g., ASD). Exposure is likely to have little effect on acceptance of the food and it is very likely that the practitioner will have to deal with additional avoidance based behaviours and strict individual rules derived from food texture and other sensory cues. Unlike the 'normal' learning-dependent versions of food refusal, these children are often not immediately happy to play with or touch foods that are either novel or have been already experienced and rejected.

\section{Fear-Based Food Refusal}

Fear-based food refusal covers a multitude of potential phobias that express themselves through the refusal to eat. A child may present with phobias about chewing, swallowing or choking on food, or may have a phobia about eating a specific food or foods. Definitions of food phobia usually refer to the intense fear of chewing, swallowing or choking on food or drink which often occurs following a significant experience where the individual has learnt, through conditioning, to fear placing specific food items in their mouth. This form of food refusal often results in an extremely restricted diet and can result in the virtual abstinence from eating solid foods and fluids ${ }^{17}$. The potential implications of this disorder for the child's health and development can be serious, particularly if the child is refusing to eat virtually all solid foods and fluids like the child described in Nock's ${ }^{17}$ paper. Food phobia is not specific to childhood and can develop at any time ${ }^{16}$, demonstrating that food phobics can have a normal 
relationship with food until they experience an event that brings about their aversion. A review by McNally ${ }^{16}$ indicates that the vast majority of those that have been diagnosed with choking phobia have other anxiety or affective disorders as part of their profile, and whilst the food phobia may result as part of a more general anxiety disorder, it is also possible that the experience of choking and the aversion to food could also exacerbate other anxieties.

Characteristics of this form of food refusal mirror other phobic behaviours with the child reacting to food with elevated levels of anxiety and fear, which can sometimes progress to the child experiencing panic attacks. Food phobia is often, but not always, associated with weight loss $^{16}$ and so these children can present as being underweight. Many of those individuals who do not lose weight may not have globalised their negative experience and aversion to all foods. For these children, their experience may have conserved some food items and thus allows them to maintain their weight through the consumption of a more limited diet. This suggests that food phobia should be seen as a behaviour that exists on a continuum. The target food that causes the anxiety may be specific to one, few or virtually all foods depending on the individual child. The more the child globalises their experience to different foods, the more important professional intervention will be. It is unlikely that a child that maintains a specific food phobia to one food item will require referral to a feeding disorder clinic, as they will be able to maintain a healthy diet through the avoidance of only the target food. Beyond the intense fear that some of the more extreme cases of food phobic children suffer from during mealtimes, they can also be differentiated from other forms of food refusal by their vigorous resistance to placing the food in the mouth, chewing it and ultimately swallowing $\mathrm{it}^{80}$. This characteristic alone makes this type of food refusal extremely difficult and slow to treat.

\section{Appetite Awareness \& Autonomy-Based Food Refusal}

Of all of the categories presented within this paper, the class of appetite-awarenessautonomy-based food refusal is the most contentious. The leading proponent of this group as a different category from other types of food refusal is that of Chatoor. Over the last twentyfive years, Chatoor has offered several research and clinical descriptions of appetiteawareness-autonomy-based food refusal under the term 'infantile anorexia'. The justification of using the term 'anorexia' within the clinical description is derived from the observations that the pathology contains similar characteristics to other sub-types of adult anorexia nervosa with the child having problems with respect to their autonomy from the primary caregiver ${ }^{21}$. Furthermore, Chatoor ${ }^{81}$ also suggests that the child's intense emotional arousal and desire to play inhibits them from attending to their nutritional needs. The diagnostic criteria for 'infantile anorexia' offered by Chatoor et $\mathrm{al}^{29}$ can be separated into infant-related and parent-related symptoms. The infant-related issues are that food refusal will: vary from meal to meal; vary depending on the person feeding them; will have a poor calorie intake for at least 2 months; weight status below the $5^{\text {th }}$ percentile for age; and no developmental delay (except for motor and expressive language development in severe cases). The parent-related components to this disorder that will lead to a diagnosis of 'infantile anorexia' are separated into their 
perceptions and their behaviour. Parent's perception of the child will be: that they have a poor appetite; overly attention-seeking and curious; and stubborn during feeding. The parent's behaviour is brought about by their elevated levels of anxiety about their child's eating behaviour and is characterised by: coaxing the infant to eat more; distracting the infant with toys to induce eating; feeding the infant at any time of the day or night; offering different foods if the infant does not eat; and force-feeding the infant.

The critical period for developing appetite-awareness-autonomy-based food refusal is suggested to be during the period of progression from spoon-feeding to self-feeding. Relative strengths of 'infantile anorexia' as a separate disorder are gained through Chatoor et $\mathrm{al}^{21,29}$ insisting that all other causes are ruled out before an official diagnosis can be met. Counterarguments to this proposition would obviously derive from the social awareness of the child at this time and the tenuous comparison to adult anorexia nervosa with the explanation that the child cannot decide whether to remain dependent on, or autonomous from, their parent. Chatoor \& Ganiban ${ }^{14}$ also suggest that appetite-awareness-autonomy-based food refusers have an inability to either register hunger, respond to hunger or to effectively communicate hunger in early life. Irrespective of the actual name of this category and the general acceptance of it, there are two important and defining factors that differentiate this form of food refusal from the others. According to Chatoor and colleagues, these two factors are the child's awareness of their own hunger and their problems with autonomy from the caregiver. The lack of awareness about hunger would draw obvious comparisons with the development of external eating in later life ${ }^{82}$, as these individuals have also been proposed to have a lack of awareness around their physiological determinants of hunger, satiation and satiety.

Children within this group may present as being underweight with moderate to severe growth faltering. Their food intake may be erratic and often additionally defined by the consumption of small amounts during a meal. These children may refuse to eat and may try to avoid mealtime interactions. The child's caregiver is likely to be extremely anxious about their eating; although there is no evidence as to whether this is a cause or effect of the child's food refusal. There have also been suggestions that autonomy based food refusal may be characterised by an insecure attachment between the child and their caregiver with the child experiencing increased separation anxiety ${ }^{14}$. The eating behaviour of these children is often erratic and unpredictable with the child accepting a particular food at one meal and rejecting it at another ${ }^{29}$. Interventions with these children will have to consider their emotional intensity and any issues between control and autonomy that may exist. In sum, the behavioural characteristics of appetite-awareness-autonomy-based food refusal are that the child may present as significantly underweight, may have temperament problems and that there may be autonomy based problems between the child and the primary caregiver. The child's diet may 
be limited in terms of both the amount consumed and dietary variety. Acceptance of food in these children is likely to be a long process and will require a more holistic intervention.

The Separation of Food Refusal Behaviours

\section{FIGURE 3 GOES HERE}

The evidence would suggest that there are distinct and separate behaviours and disorders associated with food refusal that can lead to low or even pathological levels of dietary variety. We believe that based on the available evidence there are five distinct types of food refusal that may require different intervention strategies in order to improve the child's diet and development. Figure 3 offers the theoretical postulation about what would happen to the different children's dietary variety as they develop. For the developmental refuser, their dietary variety will decrease during childhood but should rapidly increase during later childhood, adolescence and adulthood reaching an individually determined maximal level for distrust of novel foods predominantly based on their personality characteristics.

Medical complication-related food refusal is caused by the medical complaint and therefore is outside of the child's control. Such complications cover a multitude of potential complaints associated with any of the major organs or the anatomical structures involved in digestion. In such cases, the child may go through an extremely restricted and extended period of low dietary variety or will have complications with eating normally. This may then impact on the child's fine motor control leading to a delayed development in increasing their potential food choices. Upon recovery, the child may learn to accept food relatively easily; although there may be initial difficulties in acceptance because of a lack of experience which activates the natural neophobic tendencies of the child.

The selective food refuser may initially follow a similar pattern to learning-dependent food refuser during weaning but is likely to rapidly decrease their potential array of food choices during self-feeding. Their diet may be typified by extremely low variety and it may take a long period of time to accept a new food and often the food may never be accepted. Foods that may be accepted may activate sweet and salt taste receptors and thus may be reliant on ingrained genetically determined neurobiological taste preferences.

A fear-based food refuser may be dependent on a specific life experience that results in uncontrolled anxiety around eating particular food or foods. The development of their dietary variety may be in most ways normal, mirroring developmental forms of food refusal. At any point either in childhood or beyond, a choking experience, negative experience or general anxiety with a food or foods may cause this type of refuser to begin to restrict their diet which may lead to weight loss. 
Finally, the appetite-awareness-autonomy-based food refuser may not go through similar stages of weaning and the development of self-feeding in the same way as other children. Their acceptance of foods is likely to be extremely rigid incorporating factors that have nothing to do with the actual food itself. Characteristics of the dietary variety development in these children is totally absent and they are likely to present with significantly poor weight gain indicative of a diet that is not only low in variety but also low in total calories.

\section{Future Directions}

\section{TABLE 2 GOES HERE}

Intervening to improve the diet of a child who is refusing food is a complex process. Although the simple act of rejecting an offered food or foods may appear to be a homogenous behaviour, there are subtle differences that will have ramifications for the type and prospective success of the management strategies required. A summary of the key behavioural characteristics can be seen in Table 2. These refusal behaviours can also be ranked in terms of their severity, which may also be indicative of the necessary time for therapeutic intervention. All the types of food refusal behaviours discussed here are likely to require professional intervention: medical complication-related food refusal; selective food refusal; fear-based food refusal; and appetite-awareness-autonomy-based food refusal. One caveat to this classification is that even the learning-dependent (natural) food refusal may require minor interventions in those cases that lie at the extreme end of food neophobia. These cases may make up a large proportion of the children referred to feeding clinics for therapeutic intervention but it is likely that such cases will respond extremely quickly within the therapeutic setting.

More research into feeding disorders is necessary. In particular, there is a lack of quantitative research which means that the line of argument developed here is often based on qualitative differences dependent on case study understanding of the phenomenon. Much more research focus is required within this field from both a clinical description and research perspective in order to fully appreciate the most common type of 'eating' disorder. The specific problem that faces us is that the amount of specific sub-types of feeding disorders within a single clinic is often disproportionately distributed and some are too few in number to provide an adequate pool of participants for quantitative investigations. One way to overcome this issue would be to create a central repository or group that can facilitate the combination of clinical samples for quantitative investigations. Furthermore, there needs to be more research on successful treatment regimes for intervening in all types of feeding disorders. This would need to include a clinic wide decision-making process for treating children taking them from initial referral or presentation to successful treatment. Only then can the 
theoretical postulations offered by researchers be validated against separate and successful treatment strategies for the five different categories of food refusal.

\section{Conclusions}

There remain a wealth of different definitions and terms for what constitutes problems with feeding in childhood. This paper attempts to describe and delineate in more detail some of the more common problems with feeding that exist in childhood under the umbrella term of 'food refusal'. Food refusal is a common problem that if ignored can lead to medical and psychological problems. Focusing on classifying disordered eating in childhood can be a counterproductive activity which essentially misses many children who do not fit certain diagnostic criteria, focussing instead on the behaviours associated with, or common to, food refusal may help to better identify children who are in need of professional intervention. The decision to intervene is clearly a holistic process that requires attention of the medical, nutritional and psychological state of the child and family. Not only can food refusal have consequences for the child's diet, weight and nutritional status, but food refusal can also lead to a great deal of anxiety and stress for the child and their family. Interventions need to focus on the range of behaviours associated with food refusal if they are to improve the quality of life, nutritional and psychological status of these children. 


\section{REFERENCES}

[1]Hoek HW, van Hoeken D. Review of the prevalence and incidence of eating disorders. Int J Eat Disord 2003; 34: 383-96.

[2]Favaro A, Ferrara S, Santonastaso P. The spectrum of eating disorders in young women: a prevalence study in a general population sample. Psychosom Med 2004; 65: 701-8.

[3]Garfinkel PE, Lin E, Goering P, et al. Bulimia nervosa in a Canadian community sample: prevalence and comparison subgroups. Am J Psychiatry 1995; 152: 1052-8.

[4]Hudson JI, Hiripi E, Pope HG Jr, Kessler RC. The prevalence and correlates of eating disorders in the national comorbidity survey replication. Biol Psychiatry 2007; 61: 348-58

[5]Ramsay $M$, Veroff $V$. The physiology of feeding and its implication in the treatment of infant feeding disorders. Infant Behav Dev 1998; 21: 119.

[6]Coulthard H, Harris G. Early food refusal: the role of maternal mood. J Reprod Infant Psyc 2003; 21: 335-45.

[7]Dahl M, Sundelin C. Early feeding problems in an affluent society. I. Categories and clinical signs. Acta Paediatr Scand 1986; 75: 370-9.

[8]Singer LT, Ambuel B, Wade S, Jaffe AC. Cognitive-behavioral treatment of health-impairing food phobias in children. J Am Acad Child Psy 1992; 31: 847-52.

[9]Corbett SS, Drewett RF. To what extent is failure to thrive in infancy associated with poorer cognitive development? A review and meta-analysis. J Am Acad Child Psy 2004; 45: 641-54.

[10]Marchi M, Cohen P. Early childhood eating behaviors and adolescent eating disorders. J Am Acad Child Psy 1990; 29: 112-7.

[11]Dovey TM, Staples PA, Gibson EL, Halford JCG. (2008). Food neophobia and picky/fussy eating: a review. Appetite 2008; 50(2-3): 181-93.

[12]Lindberg L, Bohlin G, Hagekull B. Early feeding problems in a normal population. Int J Eat Disord 1990; 10: 395-405.

[13]Shore BA, Babbitt RL, Williams KE, Coe DA, Snyder A. Use of texture fading in the treatment of food selectivity. J Appl Behav Anal 1998; 31: 621-33.

[14]Chatoor I, Ganiban J. Food refusal by infants and young children: diagnosis and treatment. Cogn Behav Pract 2003; 10: 138-46.

[15]Kern L, Marder TJ. A comparison of simultaneous and delayed reinforcement as treatments for food selectivity. J Appl Behav Anal 1996; 29: 243-6.

[16]McNally RJ. Chocking phobia: a review of the literature. Compr Psychiat 1994; 35: 83-9.

[17]Nock MK. A multiple-baseline evaluation of treatment of food phobia in a young boy. J Behav Ther Exp Psy 2002; 33: 217-25.

[18]Paul C, Williams KE, Riegal K, Gibbons B. Combining repeated taste exposure and escape prevention: an intervention for the treatment of extreme food selectivity. Appetite 2007; 49: 708-11.

[19]Benoit D, Wang EEL, Zlotkin SH. Discontinuation of enterostomy tube feeding by behavioral treatment in early childhood: a randomised controlled trail. J Pediatr 2000; 137: 498-503. 
[20]Dellert SF, Hyams JS, Treem WR, Geertsma MA. Feeding resistance and gastroesophageal reflux in infancy. J Pediatr Gastr Nutr 1993; 17: 66-71.

[21]Chatoor I, Hirsch R, Ganiban J, Persinger M, Hamburger E. Diagnosing infantile anorexia: the observation of mother-infant interactions. J Am Acad child Psy 1998, 37, 959-67.

[23]Carruth BR, Ziegler PJ, Gordon A, Barr SI. Prevalence of 'picky/fussy' eaters among infants and toddlers and their caregivers' decision about offering new food. J Am Diet Assoc 2004; 104: S57-S64.

[24]Koivisto-Hursti U-K, Sjöden P. Food and general neophobia and their relationship with self-reported food choice: familial resemblance in Swedish families with children of ages 7-17 years. Appetite 1997; 29: 89-103.

[25]Pliner P. Development of measures of food neophobia in children. Appetite 1994; 23: 14763.

[26]Pliner P, Loewen ER. Temperament and food neophobia in children and their mothers. Appetite 1997; 28: 239-54.

[27]Harris G. In Southall A, Schwartz A. Ed, Feeding Problems in Children. Abingdon, Radcliffe. 2000; 77-88.

[28]Harris G, Blisset J, Johnson R. Food refusal associated with illness. Child Psy Psychiat Rev 2000; 5: 148-56.

[29]Chatoor I, Getson P, Menvielle E, et al. A feeding scale for research and clinical practice to assess mother-infant interactions in the first three years of life. Inf Mental HIth J 1997; 18: 76-91.

[30]Hagekull B, Dahl M. Infants with and without feeding difficulties: maternal experiences. Int J Eat Disord 1987; 6: 83-98.

[31]Galloway AT, Fiorito LM, Francis LA, Birch LL. 'Finish your soup': counterproductive effects of pressuring children to eat on intake and affect. Appetite 2006; 46: 318-23.

[32]Zajonc RB. Attitudinal effects of mere exposure [Monograph]. J Pers Soc Psychol 1968; 9: (2, Pt. 2).

[33]Rozin P. In Kroeze JHA. Ed, Preference, Behaviour \& Chemoreception. London, Information Retrieval Limited. 1979; 289-97

[34]Pliner P, Hobden K. Development of a scale to measure the trait food neophobia. Appetite 1992; 19: 105-20.

[35]Birch LL, Fischer JO. Development of eating behaviours among children and adolescents. Pediatrics 1998; 101: 539-49.

[36]Rozin P, Vollmecke T. Food likes and dislikes. Annu Rev Nutr 1998; 6: 433-56.

[37]Wardle J, Herrera M-L, Cooke LJ, Gibson EL. Modifying children's food preferences: the effects of exposure and reward on acceptance of an unfamiliar food. Eur J Clin Nutr 2003; 57 : 341-8.

[38]Addessi E, Galloway AT, Visalberghi E, Birch LL. Specific social influences on the acceptance of novel foods in 2-5-year-old children. Appetite 2005; 45: 264-71.

[39]Cashdan E. A sensitive period for learning about food. Hum Nature 1994; 5: 279-91. 
[40]Cooke L, Wardle J, Gibson EL. Relationship between parental report of food neophobia and everyday food consumption in 2-6-year-old children. Appetite 2003; 41: 205-6.

[41]Koivisto U-K, Sjöden P. Food and general neophobia in Swedish families: Parent-child comparisons and relationships with serving specific foods. Appetite 1996; 26: 107-18.

[42]Nicklaus S, Boggio V, Chababnet C, Issanchou S. Prospective study of food variety seeking in childhood, adolescence and early adult life. Appetite 2005; 44: 289-97.

[43]Rigal N, Frelut M-L, Monneuse M-O, Hladik C-M, Simmen B, Pasquet P. Food neophobia in the context of a varied diet induced by a weight reduction program in massively obese adolescents. Appetite 2006; 46: 207-14.

[44]Galloway AT, Lee Y, Birch LL. Predictors and consequences of food neophobia and pickiness in children. J Am Diet Assoc 2003; 103: 692-8.

[45]Loewen R, Pliner P. Effects of prior exposure to palatable and unpalatable novel foods on children's willingness to taste other novel foods. Appetite 1999; 32: 351-66.

[46]McCrae RR, Costa PT Jr, Terracciano A, et al. Personality trait development from age 12 to age 18: Longitudinal, cross-sectional, and cross-cultural analyses. J Pers Soc Psychol 2002; 83: 1456-68.

[47]Steptoe A, Pollard TS, Wardle J. Development of a measure of motives underlying the selection of food: The food choice questionnaire. Appetite 1995; 25: 267-84.

[48]Birch LL, Fischer JO. Appetite and eating behaviour in children and adolescents. Pediatrics 1995; 101: 539-49.

[49]Wardle J. Parental influences on children's diets. P Nutr Soc 1995; 54: 747-58.

[50]Cullen KW, Rittenberry L, Olvera N, Baranowski T. Environmental influences on children's diets: results from focus groups with African-, Euro- and Mexican-American children and their parents. Health Educ Res 2000; 15: 581-90.

[51]Cullen KW, Baranowski T, Rittenberry L, Cosart C, Hebert D, de Moor C. Child-reported family and peer influences on fruit, juice and vegetable consumption: reliability and validity of measures. Health Educ Res 2001; 16: 187-200.

[52]Visalberghi E, Addessi E. (2000). Seeing group members eating a familiar food enhances the acceptance of novel foods in capuchin monkeys. Anim Behav 2000; 60: 69-76.

[53]Budd KS, McGraw TE, Farbisz R, et al. Psychosocial concomitants of children's feeding disorders. J Pediatr Psychol 1992; 17: 81-94.

[54]Field D, Garland M, Williams K. Correlates of specific childhood feeding problems. J Paediatr Child H 2003; 39: 299-304.

[55]Brant CQ, Stanich P, Ferrari AP Jr. Improvement in children's nutritional status after enteral feeding by PEG: an interim report. Gastrointest Endosc 1999; 50: 183-8.

[56]Colomb, V. Nutrition en cancérologie: aspects pédiatriques/Nutrition and cancer in children. Nutrition Clinique et Métabolisme 2001; 15: 325-34.

[57]Marin OE, Glassman MS, Schoen BT, Caplan DB. Safety and efficacy of percutaneous endoscopic gastrostomy in children. American Journal of Gastroenterology 1994; 89: 357-61.

[58]Rogers B. Feeding method and health outcomes of children with cerebral palsy. J Pediatr 2004; 145: S28-S32. 
[59]Schwarz SM, Corredor J, Fisher-Medina J, Cohen J, Rabinowitz S. Diagnosis and treatment of feeding disorders in children with developmental disabilities. Pediatrics 2001; 108: $671-6$.

[60]Veenker E. Enteral Feeding in Neurologically Impaired Children with Gastroesophageal Reflux: Nissen Fundoplication and Gastrostomy Tube Placement Versus Percutaneous Gastrojejunostomy. J Pediatr Nurs 2008; 23: 400-4.

[61]Holden CE, Puntis JWL, Charlton CPL, Booth IW. Nasogastric feeding at home: acceptability and safety. Arch Dis Child 1991; 66: 148-51.

[62]Heyman MB, Harmitz P, Acres M, et al. Economic and psychologic costs for maternal caregivers of gastrostomy-dependent children. J Pediatr 2004; 145: 551-61.

[63]Daveuly W, Guimber D, Mention K, et al. Home enteral nutrition in children: an 11-year experience with 416 patients. Clin Nutr 2005; 24: 48-54.

[64]Metheny NA, Schallom ME, Edwards SJ. Effects of gastrointestinal motility and feeding tube site on aspiration risk in critically ill patients: a review. Heart Lung 2004; 33: 131-45.

[65]Malcolm A, Thumshirn MB, Camilleri M, Williams DE. Rumination Syndrome. Mayo Clin Proc 1997; 72: 646-52.

[66]Tack J, Talley NJ, Camilleri M, et al. Functional gastroduodenal disorders. Gastroenterology 2006; 130: 1466-79.

[67]Andrassy R, Chwals WJ. Nutritional support of the pediatric oncology patient. Nutrition 1998; 14: 124-9.

[68]Bernstein IL, Webster MM. Learned taste aversion in humans. Physiol Behav 1980; 25: 363-6.

[69]Skolin I, Koivisto-Hursti U-K, Wahlin YB. Parents' perceptions of their child's food intake after the start of chemotherapy. J Pediatr Onc Nurs 2001; 18: 124-36.

[70]Galloway AT, Fiorito L, Lee Y, Birch LL. Parental pressure, dietary patterns and weight status among girls who are "picky/fussy' eaters'. J Am Diet Assoc 2005; 105: 541-8.

[71]Bosaeus I. Fibre effects on intestinal functions (diarrhoea, constipation and irritable bowel syndrome). Clin Nutr 2004; 1(2): S33-S38.

[72]Smith AM, Roux S, Naidoo NTR, Venter DJL. Food choices of tactile defensive children. Nutrition 2005; 21: 14-9.

[73]Wilbarger P. Sensory defensiveness and related social/emotional and neurological disorders. South Africa, SAISI Port Elizabeth. 2000.

[74]Schwarz SM. Feeding disorders in children with developmental disabilities. Infant Young Child 2003; 16: 373-8.

[75]Cumine V, Leach J, Stevenson G. Autism in the Early Years. London, David Fulton. 2000.

[76]Iwata BA, Riordan MM, Wohl MK, Finney JW. In Accardo, P. J. (Ed.). Failure to Thrive in Infancy and Early Childhood. Baltimore MD, University Park. 1982; 297-329.

[77]Wacker DP, Harding J, Cooper LJ, \& Derby KM. The effects of meal schedule and quantity on problematic behaviors. J Appl Behav Anal 1996; 29: 79-87. 
[78]Nicholas JS, Charles JM, Carpenter LA, King LB, Jenner W. Prevalence and characteristics of children with autism spectrum disorders in South Carolina. Ann Epidemiol 2007; 17: 747-8.

[79]Signoretta S, Maremmani I, Liguori A, Perugi G, Akiskald HS. Affective temperament traits measured by TEMPS-I and emotional-behavioral problems in clinically-well children, adolescents, and young adults. J Affect Disorders 2005; 85: 169-80

[80]Chatoor I. Ganiban J, Harrison J, Hirsch R. The observation of feeding in the diagnosis of the posttraumatic feeding disorder of infancy. J Am Acad Child Psy 2001; 40: 595-602.

[81]Chatoor, I. Infantile anorexia nervosa: a developmental disorder of separation and individuation. J Am Acad Psychoan 1989; 17: 43-64.

[82]Schachter S. Obesity and eating. Science 1968; 16: 751-6. 
Table 1. DSM-IV diagnostic criteria for Feeding Disorders of Infancy or Early Childhood
A. Feeding disturbance as manifest by persistent failure to eat adequately with significant failure to gain weight or significant loss of weight over at least 1 month.
B. The disturbance is not due to an associated gastrointestinal or other general medial condition (e.g. esophageal reflux).
C. The disturbance is not better accounted for by another mental disorder (e.g. Rumination Disorder) or by lack of available food.
D. The onset is before age 6 years.

Table 2 Summary table for the behavioural characteristics associated with the different terms presented in this paper - see attached word document

Figure 1. Previous categorisation of terms used in children's food refusal (Chatoor et $\left.\mathrm{al}^{14}\right)$ see attached powerpoint document

Figure 2. Categorisation of terms used in children's food refusal proposed in this paper - see attached powerpoint document.

Figure 3. A theoretical differentiation of habitual dietary variety between the five categories proposed in this paper - see attached powerpoint document. 

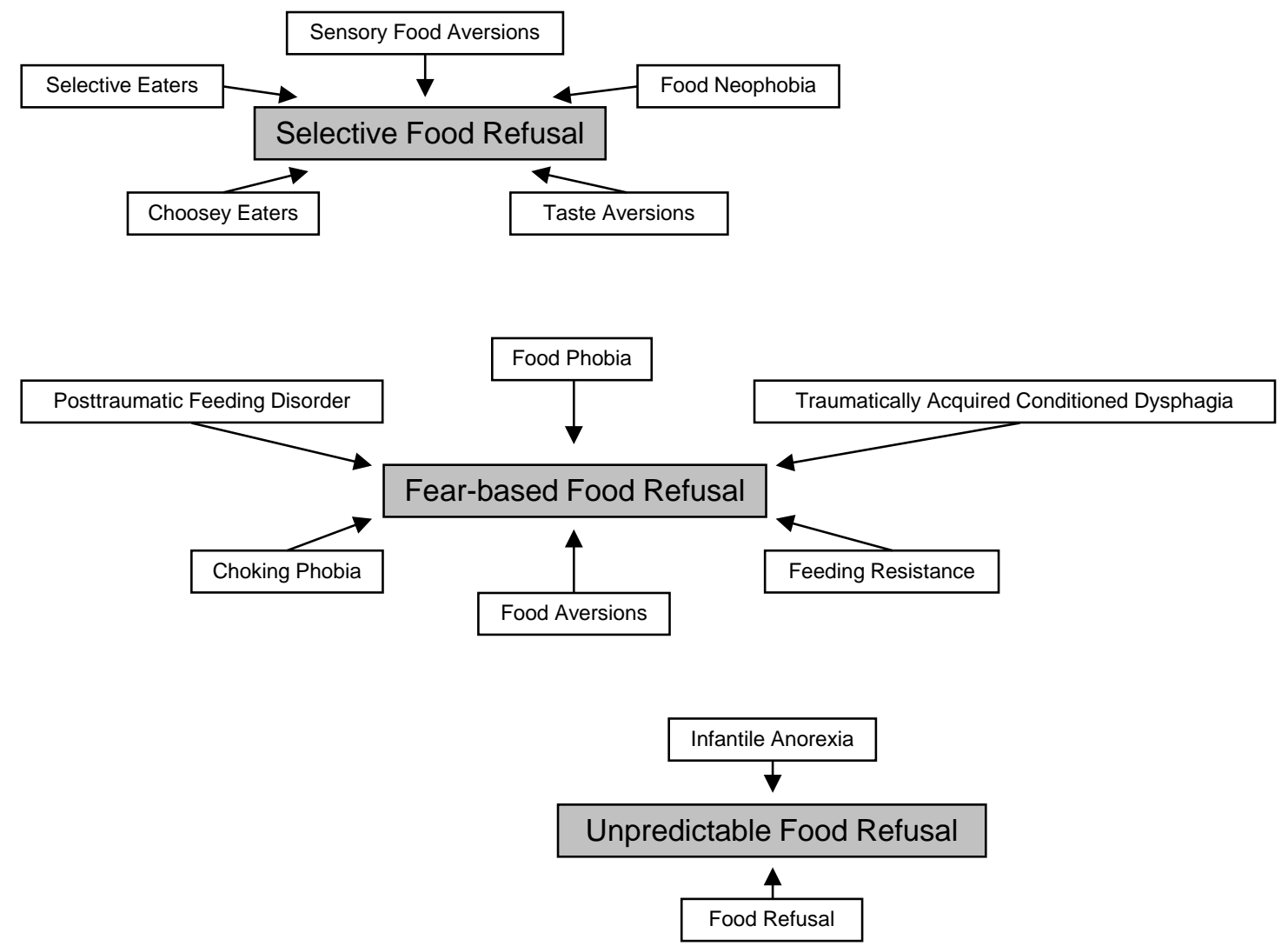


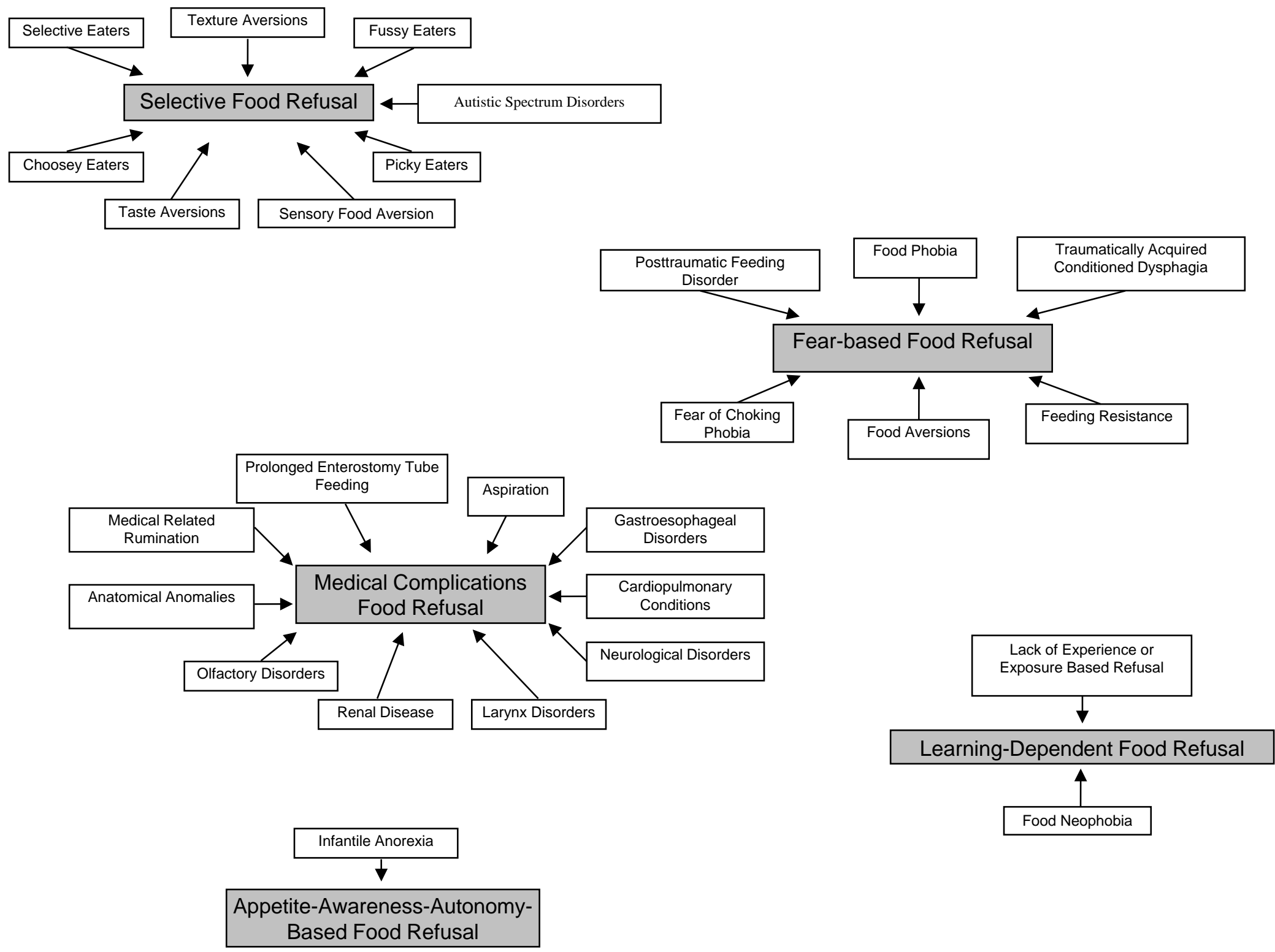




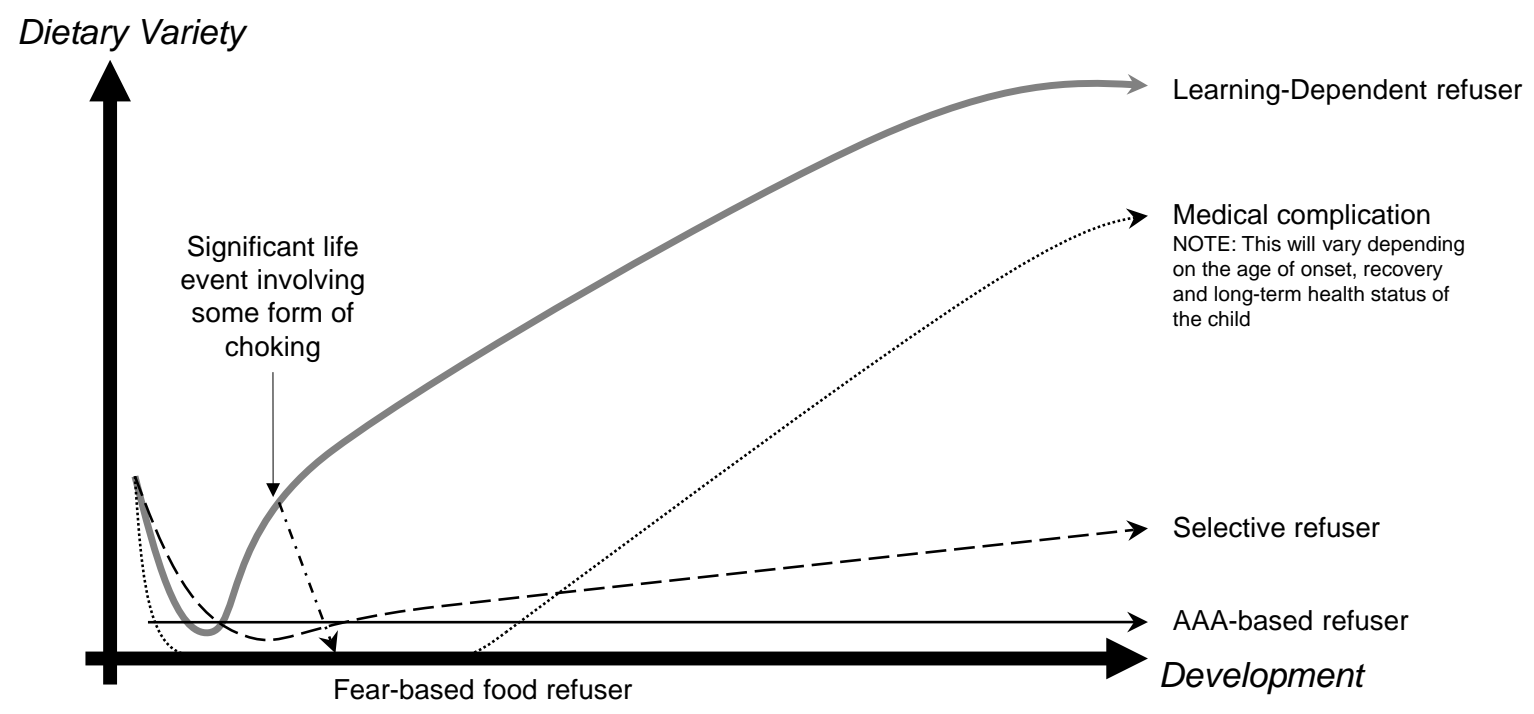

